\title{
Variations in the metabolome in response to disease activity of rheumatoid arthritis
}

\author{
Zuzana Tatar ${ }^{1 *}$, Carole Migne ${ }^{2}$, Melanie Petera ${ }^{2}$, Philippe Gaudin ${ }^{3}$, Thierry Lequerre ${ }^{4}$, Hubert Marotte ${ }^{5,6}$, \\ Jacques Tebib ${ }^{7}$, Estelle Pujos Guillot ${ }^{2}$ and Martin Soubrier ${ }^{1}$
}

\begin{abstract}
Background: Anti-Tumor Necrosis Factor (TNF) therapies are able to control rheumatoid arthritis (RA) disease activity and limit structural damage. Yet no predictive factor of response to anti-TNF has been identified. Metabolomic profile is known to vary in response to different inflammatory rheumatisms so determining it could substantially improve diagnosis and, consequently, prognosis.

The aim of this study was to use mass spectrometry to determine whether there is variation in the metabolome in patients treated with anti-TNF and whether any particular metabolomic profile can serve as a predictor of therapeutic response.

Methods: Blood samples were analyzed in 140 patients with active RA before initiation of anti-TNF treatment and after 6 months of Anti-TNF treatment (100 good responders and 40 non-responders). Plasma was deproteinized, extracted and analyzed by reverse-phase chromatography-QToF mass spectrometry. Extracted and normalized ions were tested by univariate and ANOVA analysis followed by partial least-squares regression-discriminant analysis (PLS-DA). Orthogonal Signal Correction (OSC) was also used to filter data from unwanted non-related effects. Disease activity scores (DAS 28) obtained at 6 months were correlated with metabolome variation findings to identify a metabolite that is predictive of therapeutic response to anti-TNF.
\end{abstract}

Results: After 6 months of anti-TNF therapy, 100 patients rated as good responders and 40 patients as non-responders according to EULAR criteria. Metabolomic investigations suggested two different metabolic fingerprints splitting the good-responders group and the non-responders group, without differences in anti-TNF therapies. Univariate analysis revealed 24 significant ions in positive mode $(p<0.05)$ and 31 significant ions in negative mode $(p<0.05)$. Once intersected with PLS results, only 35 ions remained. Carbohydrate derivates emerged as strong candidate determinants of therapeutic response.

Conclusions: This is the first study describing metabolic profiling in response to anti-TNF treatments using plasma samples. The study highlighted two different metabolic profiles splitting good responders from non-responders.

Keywords: Rheumatoid arthritis, Metabolomics, Anti-TNF-alpha therapy, Drug response biomarkers

Abbreviations: ANOVA, Analysis of variance; Anti-CCP, Anti-cyclic citrullinated peptide; CRP, C-reactive protein; DAS 28, Disease activity scores; ESR, Erythrocyte sedimentation rate; EULAR, European League Against Rheumatism; HMDB, Human metabolome database; IgG, Immunoglobulin G; NMR, Nuclear Magnetic Resonance; OSC, Orthogonal Signal Correction; PLS-DA, Partial least-squares regression-discriminant analysis; QTOF, Quadrupole-time-of-flight; RA, Rheumatoid arthritis; RT, Retention time; SD, Standard deviation; TNF, Tumor Necrosis Factor; VIP, Variable importance in the projection

\footnotetext{
* Correspondence: ztatar@chu-clermontferrand.fr

'Rheumatology Department, CHU Gabriel Montpied, 58 rue Montalembert

B.P. 392, 63011 Clermont-Ferrand, France

Full list of author information is available at the end of the article
} 


\section{Background}

Anti-Tumor Necrosis Factor (TNF) drug therapies were first introduced in rheumatoid arthritis (RA) treatment more than 10 years ago. Anti-TNF agents are able to control RA disease activity and limit structural damage. However, a third of patients do not respond to treatment, prompting efforts to find predictive factors of therapeutic response in order to optimize patient management and to limit the systematic use of these drugs. Since the combination of potential side effects like infections, reactivation of tuberculosis and basocellular carcinomas, with high cost (averaging $€ 12000$ per year per patient) can often outweigh the therapeutic benefits. Despite this effort, no predictive factor of response to anti-TNF has yet been clearly identified [1-3].

The metabolome, i.e. the complete set of metabolites, varies in response to different diseases, and so can potentially make diagnosis and treatment easier. Metabolomic profile is known to vary in response to different inflammatory rheumatisms, so determining it could substantially improve diagnosis and, consequently, prognosis [4]. In patients with inflammatory rheumatism, studying the metabolome could facilitate earlier diagnosis of rheumatoid arthritis (RA) and subsequently better management and prognosis.

The aim of this study was to determine whether there is variation in the metabolome in patients treated with anti-TNF and whether any particular metabolomic profile can serve as a predictor of therapeutic response.

\section{Methods}

\section{Study population}

All RA patients were aged $>18$ years and met the 1987 revised classification criteria of the American College of Rheumatology [5]. All patients has failed to respond to treatment with at least one disease-modifying antirheumatic drug, and were treated with methotrexate at a dose of at least $7.5 \mathrm{mg}$ weekly. Prednisolone was allowed provided the dose remained stable and did not exceed $10 \mathrm{mg}$ daily.

Patients with RA requiring anti-TNF-alpha treatment were recruited in the Rheumatology department of university teaching hospitals at Clermont-Ferrand, St-Etienne, Lyon, Rouen, and Grenoble, all in France. A plasma bank was created from samples taken from every patient. Anticitrullinated protein antibodies (ACPA) were detected with enzyme-linked immunoabsorbent assay (ELISA) and were considered positive at a serum concentration $\geq 5 \mathrm{IU} /$ ml. Rheumatoid factor (RF) was measured by the particleenhanced immunonephelometry with the lower level of detection of $10 \mathrm{IU} / \mathrm{ml}$.

Here we chose to consider only good-responders and non-responders in order to increase the chances of finding significant differences in metabolic profiles. Indeed, only baseline blood samples (collected before the initiation of anti-TNF treatment : "M0") of good-responders and non-responders were used for the metabolomics analysis. In total, 140 venous plasma samples were withdrawn, in a non-fasting state, before the initiation of anti-TNF-alpha treatment (M0) and frozen at $-80{ }^{\circ} \mathrm{C}$ until analysis.

RA patients received anti-TNF therapy (infliximab, abatacept or etanercept) and this treatment was kept unchanged for at least the first 6 months. Initially, all the patients had active RA at M0 (DAS-28 >3.2) requiring biological therapy and underwent disease severity assessments (DAS-28 ESR, DAS-28 CRP, Health Assessment Questionnaire scores [6]). Anti-TNF was started at usual doses and by standard administration. Three different anti-TNF therapies were used : infliximab, adalimumab or etanercept. Six months after initiation of treatment (M6), DAS28 was assessed to classify therapeutic response according to EULAR criteria [7] and thus create three groups: good responders, moderate responders, and non-responders. A good clinical response in RA was defined as a DAS28 $\leq 3.2$ and a $>1.2$-point improvement in DAS28 after anti-TNF therapy [8]. A non-response in RA was defined as a DAS28 $\geq 5.1$ and a $<0.6$-point improvement in DAS28 after the anti-TNF-alpha therapy [8].

Disease activity scores obtained at M6, and metabolome variation findings were cross-correlated to identify a metabolomics fingerprint that is predictive of therapeutic response to anti-TNF-alpha therapy.

The study was approved by the appropriate institutional review boards/independent ethics committees (Committee of Patient's Protection Grenoble 05-CHUG3 et Sud-Est 6 -AU731), and was carried out in accordance with the ethical principles of the Declaration of Helsinki. All patients gave written, informed consent.

\section{Metabolomics analysis}

The blood samples were first deproteinized and extracted, then analyzed by reverse-phase liquid chromotographyelectrospray QToF mass spectrometry. The metabolic profiles were collected using positive and negative ionization, in full-scan mode, on a mass range from 90 to $1000 \mathrm{~m} / \mathrm{z}$. The data were then centroided and corrected before reprocessing to obtain a matrix containing retention times, exact masses and intensities of potential markers.

A preliminary quality analysis of the data performed by principal component analysis detected the possible effect of fouling of the ionization source of the mass spectrometer during the sample injection series. These effects were checked and corrected using quality control samples corresponding to the pools of all the analyzed samples. After linear regression modeling of this instrumental drift on quality control samples, the model thus obtained was used to correct all extracted ion intensities. 
The list of extracted and normalized ions was considered in univariate analysis. The results from metabolomic investigations were analyzed by ANOVA and cross-compared against partial least-squares regression-discriminant analysis data (PLS-DA). Orthogonal Signal Correction (OSC) was also used to filter data from unwanted non-related effects. Results were considered statistically significant if $p<0.05$ and if VIP (Variable Importance of the Projection) $>1.5$. Ions were identified by annotation in the HMDB database.

\section{Statistical analysis}

This was an exploratory and multicentric study. Biostatistical analyses followed the design of a case-control study with binary analysis ("case" subjects are goodresponders according to EULAR response criteria; "control" subjects are non-responders according to EULAR response criteria).

The justification for the number of subjects was based primarily on ability to recruit, sample sizes of princeps publications, and the experience of the services involved in this research. In order to identify predictive metabolites of therapeutic response, it was necessary to include 120 patients (for a bilateral risk of error $\alpha$ equal to $5 \%$ and a power $\geq 90 \%$ ) considering:

1) an in-population response rate of $60 \%$ : distribution was $70 \%$ responders (breaking down as $35 \%$ very good responders and 35 moderate responders) and $30 \%$ non-responders;

2) the rules for determining the required number of subjects involved in the study of predictors of a binary response variable as defined by Harrell et al. [9].

Concerning power estimation for each metabolite, we explored different simulations, as proposed by Cohen
[10], using as starting point the conventional effect sizes (ES) for the Student test: small $(E S=0.2)$, mean $(E S=0.5)$ and high $(E S=0.8)$. Given the sample size of this study (i.e. 70 cases and 50 controls), the expected ES was 0.6 for $\alpha=5 \%$ and $\beta=10 \%$.

Statistical analyses were performed in STATA v11 (STATA Corp, College Station, TX). Numerical outcomes were expressed as mean \pm SD (for Gaussian distributions). Nominal outcomes were expressed as raw values and percentages. Level of significance was set at $5 \%$.

\section{Results}

After 6 months of anti-TNF therapy, 100 patients were considered good-responders and 40 patients as nonresponders according to EULAR criteria [7]. The baseline characteristics of the patients are summarized in the Table 1. There was no difference in response to anti_TNF treatment between patients with or without rheumatoid factors or anti-citrullinated peptide.

Metabolomic investigations suggested two different metabolic fingerprints segregating good-responders group from non-responders group (Fig. 1). There is a concrete global effect of discriminative ions, even though we were unable to report any single discriminating biomarker that could simplify routine management of RA.

The univariate analyses revealed 24 significant ions in positive mode $(p<0.05)$ and 31 significant ions in negative mode $(p<0.05)$. Once intersected with PLS results, only 35 ions remained. The ions-of-interest were then identified by querying the HMDB database (Table 2). Carbohydrate derivates (D-glucose, D-fructose, sucrose, and maltose) emerged as determinants of therapeutic response. Because of lack of pertinent biomarkers, no absolute quantifications and identifications of different metabolites were proceeded.

Table 1 Baseline characteristics of rheumatoid arthritis patients by response to anti-TNF therapy at 6 months

\begin{tabular}{llll}
\hline & Good responders $(n=100)$ & Non-responders $(n=40)$ & $P$-value \\
\hline Age, years (mean \pm SD) & $50 \pm 14$ & $57 \pm 13$ & 0.44 \\
Female (\%) & $76(76)$ & $31(77.5)$ & 1.00 \\
Disease duration (months, range) & $9(3.3-15.3)$ & $10(3.7-12.8)$ & 0.54 \\
Infliximab (\%) & $13(13)$ & $7(17)$ & $17(43)$ \\
Adalimumab (\%) & $29(29)$ & $16(40)$ & 0.64 \\
Etanercept (\%) & $58(58)$ & $5.0 \pm 1.2$ & 0.32 \\
DAS-28 M0 (Mean \pm SD) & $4.8 \pm 0.9$ & $4.8 \pm 1.2$ & - \\
DAS-28 M6 (Mean $\pm S D)$ & $2.2 \pm 0.6$ & 70 & 0.82 \\
RF positivity (\%) & 77.4 & 62 & 0.25 \\
ACPA positivity (\%) & 75 & $35 \pm 23$ & 0.18 \\
ERS (mm/h) & $32 \pm 25$ & $21 \pm 18$ & 0.37 \\
CRP (mg/l) & $17 \pm 19$ & 31 & 0.45 \\
Bone erosion (\%) & 38 & 0.37 \\
\hline
\end{tabular}

$R F$ rheumatoid factor, $A C P A$ anticitrullinated protein antibodies, ERS erythrocyte sedimentation rate, $C R P$ C-reactive protein 
a
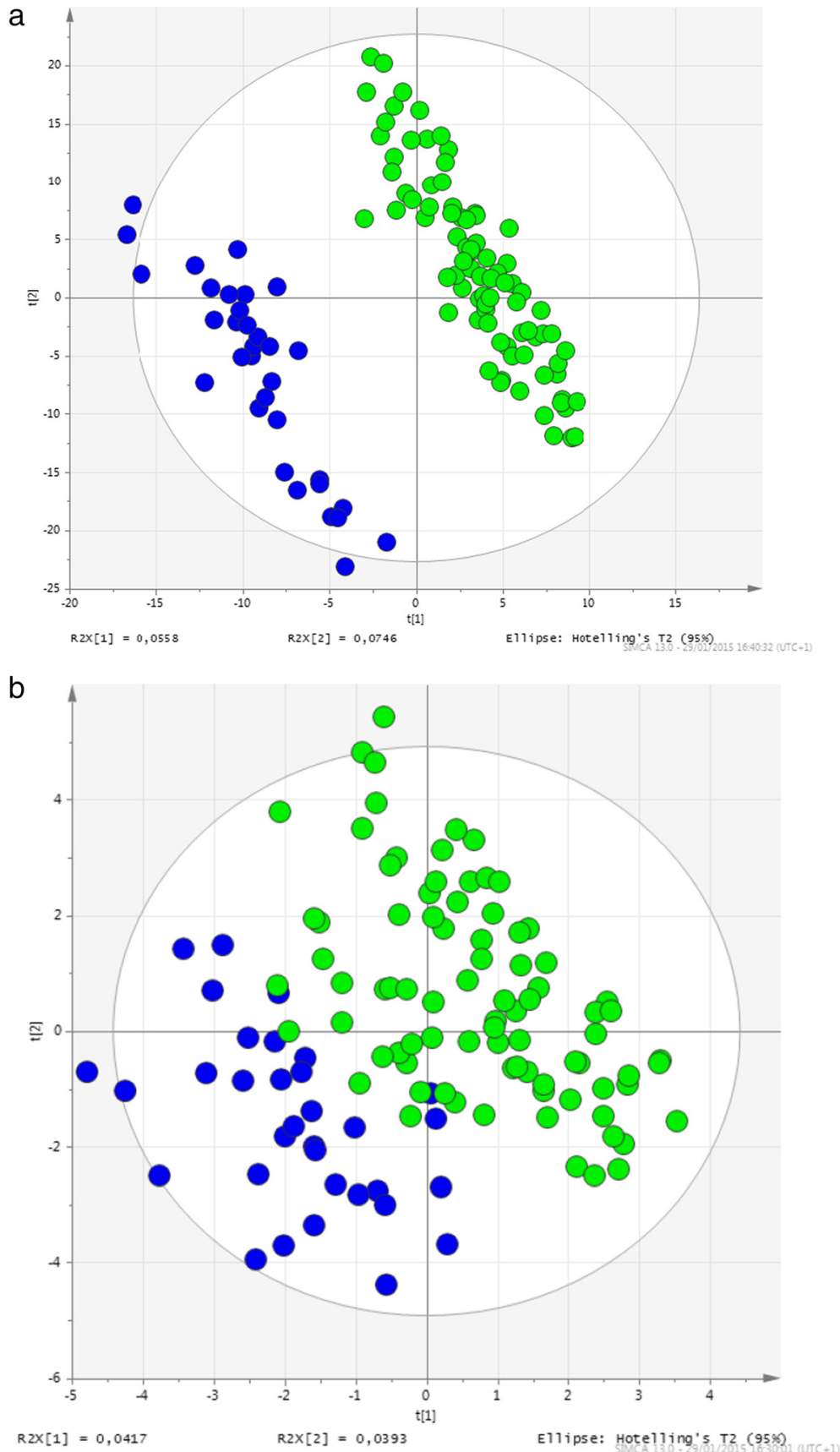

Fig. 1 Metabolomic fingerprinting distinguished between baseline plasma samples from RA patients demonstrating good response to anti-TNF agents (green circles) and no response (blue circles) at 6 months in positive (a) and negative (b) mode analysis

Despite of the discriminatory power of ions, the construction of a PLS model based only on the most significant ions did not allow sufficient discrimination: only a global effect could be linked to the therapeutic response.

There was no difference between different groups of anti-TNF therapies (infliximab, etanercept and adalimumab. We found no interaction of the metabolome with age, sexe and rheumatoid factors or anti-citrullinated peptide status, use of steroids or methotrexate. We have no sufficient data about smoking status, glucose blood levels or lipidic profile.

\section{Discussion}

This is the first study to describe metabolomic profiling in response to anti-TNF treatments using plasma samples. Our study highlighted two different metabolomic 
Table 2 Baseline plasma metabolites most strongly correlated with response to TNF antagonists using partial least-squares regression and ANOVA (VIP $>1$ with $p<0.1$ and VIP $>1.5$ with $p<0.05)$

\begin{tabular}{|c|c|c|}
\hline Ion mass $(\mathrm{Da})$ & $\mathrm{RT}(\min )$ & Formula \\
\hline \multicolumn{3}{|c|}{ Positive-mode analysis } \\
\hline 181.070 & 5.2 & $\begin{array}{l}\text { C6H12O6, C42H44FeN8O8S2R4, C6H11O6R, } \\
\text { CH2NOR, NH2R, C10H17O10PR2, C5H9O5R, } \\
\text { C38H37FeN6O6SR2, C7H8N4O2, }\end{array}$ \\
\hline 224.129 & 4.2 & $\mathrm{C} 12 \mathrm{H} 17 \mathrm{NO} 3$ \\
\hline 268.148 & 12.7 & $\mathrm{C} 16 \mathrm{H} 17 \mathrm{~N} 3 \mathrm{O}$ \\
\hline 460.293 & 6.0 & $\mathrm{C} 29 \mathrm{H} 37 \mathrm{~N} 3 \mathrm{O} 2$ \\
\hline 481.350 & 13.2 & $\mathrm{C} 28 \mathrm{H} 48 \mathrm{O} 6$ \\
\hline 482.359 & 13.3 & $\mathrm{C} 24 \mathrm{H} 52 \mathrm{NO} 6 \mathrm{P}$ \\
\hline 583.257 & 9.9 & $\mathrm{C} 33 \mathrm{H} 34 \mathrm{~N} 4 \mathrm{O} 6$ \\
\hline \multicolumn{3}{|c|}{ Negative-mode analysis } \\
\hline 115.038 & 4.6 & $\mathrm{C} 5 \mathrm{H} 8 \mathrm{O} 3$ \\
\hline 128.033 & 1.4 & $\mathrm{C} 5 \mathrm{H} 7 \mathrm{NO} 3, \mathrm{C} 5 \mathrm{H} 12 \mathrm{O} 12 \mathrm{P} 3 \mathrm{R}$ \\
\hline 181.054 & 5.5 & $\mathrm{C} 9 \mathrm{H} 10 \mathrm{O} 4$ \\
\hline 203.022 & 0.9 & $\mathrm{C} 7 \mathrm{H} 8 \mathrm{O} 7, \mathrm{C} 6 \mathrm{H} 10 \mathrm{NO} 3 \mathrm{SR}, \mathrm{C} 12 \mathrm{H} 9 \mathrm{ClO}$ \\
\hline 221.156 & 12.9 & $\mathrm{C} 14 \mathrm{H} 22 \mathrm{O} 2$ \\
\hline 249.115 & 10.3 & $\mathrm{C} 14 \mathrm{H} 18 \mathrm{O} 4$ \\
\hline 281.249 & 16.3 & $\mathrm{C} 18 \mathrm{H} 34 \mathrm{O} 2$ \\
\hline 285.183 & 7.8 & $\mathrm{C} 19 \mathrm{H} 26 \mathrm{O} 2, \mathrm{C} 14 \mathrm{H} 26 \mathrm{~N} 2 \mathrm{O} 4, \mathrm{C} 19 \mathrm{H} 37 \mathrm{~N} 5 \mathrm{O} 7$ \\
\hline 313.067 & 1.2 & $\mathrm{C} 17 \mathrm{H} 14 \mathrm{O} 6$ \\
\hline 335.223 & 11.3 & $\begin{array}{l}\mathrm{C} 20 \mathrm{H} 32 \mathrm{O} 4, \mathrm{C} 10 \mathrm{H} 18 \mathrm{O} 13 \mathrm{P} 2 \mathrm{R} 2, \mathrm{C} 2 \mathrm{OH} 34 \mathrm{NO} 15 \mathrm{R}, \\
\mathrm{C} 6 \mathrm{H} 13 \mathrm{NO} 7 \mathrm{PR}, \mathrm{C} 7 \mathrm{H} 13 \mathrm{NO} 9 \mathrm{PR}, \\
\mathrm{C} 10 \mathrm{H} 15 \mathrm{NO} 11 \mathrm{PR} 3, \mathrm{C} 4 \mathrm{H} 7 \mathrm{O} 7 \mathrm{PR} 2,\end{array}$ \\
\hline 341.111 & 1.3 & $\begin{array}{l}\text { C12H22O11, C24H39N7O18P3SR, } \\
\text { C27H40N10O23P4R, C10H14N5O8P, } \\
\text { C17H24N5O10P, C8H6NO3R, NH2R }\end{array}$ \\
\hline 425.289 & 6.7 & $\mathrm{C} 2 \mathrm{OH} 38 \mathrm{~N} 6 \mathrm{O} 4$ \\
\hline 466.296 & 12.0 & $\mathrm{C} 29 \mathrm{H} 41 \mathrm{NO} 4$ \\
\hline 87.009 & 1.3 & $\mathrm{C} 3 \mathrm{H} 4 \mathrm{O} 3$ \\
\hline
\end{tabular}

$R T$ retention time, VIP variable importance in the projection

profiles splitting the good-responders group from the non-responders group. There is a concrete global effect of discriminative ions.

The major limit of our study is the impossibilty to identify any particular biomarker. However, this is an original study and despite our originality we could not found discriminant biomarkers in our patient's sample. To our knowledge, there is no other study published with more significant results.

Choice of sample is a fundamental and potentially decisive factor: indeed, analyses of blood or plasma can prove more complicated than metabolomic investigations with urine samples as they contain all the metabolites from different whole-body pathways. Opting for urine analysis could simplify metabolomic measurement and results interpretation in further research [11]. Nevertheless, metabolomic fingerprinting offers new prospects for finding predictive biomarkers of response to biological agents.

An other limit of our study is the absence of negative controls. Indeed, metabolomic approach limits the number of different samples that can be analysed at the same time and our objective was to find metabolomic differences between good and no responders. We choose to analyse more patients than negative controls in order to show more powerful results.

Contrary to other publications, the main metabolomic differences between responder and non-responder groups concerned carbohydrate derivatives. Nevertheless, recent advances in glycomics and glycol biomarker profiling show direct or indirect associations between glycosylation modifications and autoimmune disturbances in RA: peptide epitope/glycol epitope cross-reactivity, neo-expression of normally-restricted glycans, sugar induction of inappropriate processing and presentation of self-antigens to T-lymphocytes and conformational glycomodification leading to unmasking of antigenic epitopes [12].

Indeed, immune response can be strongly modulated by induced changes in glycosylation site (galactosylation, sialylation or fucosylation) in the constant domain of the IgG Fc region [13]. Animal and human studies suggest that aberrant glycosylation of IgG plays a key role in RA pathophysiology [14-16]. For example, increased fucosylation despite low galactose levels of the IgG Fc region strongly modifies antibody binding capacities and induces abnormal inflammatory reaction [14, 17]. Moreover, increased rate of glycosylated IgG was correlated with 10-year structural prognosis of RA diagnosis with $95 \%$ specificity and $90 \%$ sensitivity when associated with rheumatoid factor [14]. Glycomodifications have even distinguished early RA from other rheumatic diseases [16]. Also, Newkirk et al. reported that the rheumatoid factor avidity was significantly correlated with the presence of the glycoform of IgG lacking galactose in both circulating and immune complexes-derived IgG [18].

Glyco-biomarkers also reflect RA activity and prognosis as they are correlated with rheumatoid factor, tender joint score, and frequency of subcutaneous nodules as well as structural damage [19-22]. Moreover, levels of glycosylated IgG decreased and became normalized with anti-TNF treatment [23] or with acquired remission [24]. These results are especially consistent and coherent with our findings.

Finding predictive factors of response to biological therapy is central to the management of RA patients, both in the short term to enable rapid relief of pain and in the long term as it is now established that persistence of RA disease activity is correlated with structural damage that can lower the functional prognosis [25-27]. The potential value of the metabolome in the diagnosis of 
chronic inflammatory rheumatic diseases has been investigated in animal models and in human subjects. Analysis in the RA murine model (K/BxN transgenic mouse) [28] found that the specific metabolomic profile (nucleic acids, amino acids, reactive derivatives of oxygen, fatty acids, and the enzymes involved in lipolysis and methylation) was significantly different from that in control mice $(p=0.00075)$, and evidenced 18 metabolites out of the 59 initially studied.

In humans, Madsen et al. [29] showed the value of the metabolome in the early diagnosis of RA established even before expression of the anti-CCP antibodies. Here, mass spectrometry analysis of the metabolome established RA diagnosis with a sensitivity of $93 \%$ and a specificity of $70 \%$. The metabolomic profile in RA patients compared to healthy controls showed an increase in certain biomarkers (3-phospho-glyceric acid, d-ribofuranose and hypoxanthine) and a decrease in others (histidine, threonic acid, threonine, methionine, cholesterol and asparagine).

The metabolome appears to vary in response to RA disease activity. Lauridsen et al. [30] studied the serum metabolome by NMR mass spectrometry in 47 RA patients ( 23 with active RA and 24 with RA in remission) and 51 control subjects for one year, and reported a significant difference $(p=0.0007)$ in metabolomic profile between patients with active RA and those in remission. They identified several potential markers of RA disease severity, including total cholesterol, lactates, acetyl glycoprotein and lipid derivatives. The difference between the two groups was non-significant $(p=0.91)$ at 31 days after initiation of effective treatment in patients with active RA. The metabolome of the two patient groups remained different from that of the control group.

Nevertheless, studying the metabolome could not only improve the diagnosis of a disease but also predict the tolerance and effectiveness of certain treatments [31]. For example, analysis of the urinary metabolome was able to predict the digestive toxicity of non-steroidal inflammatory drugs in rats [32].

However, there is only one documented report on urine metabolomic variations in RA patients on anti-TNF-alpha treatment. Kapoor et al. [11] showed that variations in metabolomic profile correlated with response to anti-TNF agents according to EULAR criteria with a good sensitivity $(88.9 \%)$ and specificity (85.7\%). Variation of expression of histamine, glutamine, xanthurenic acid and ethanolamine were particularly significant and predictive. There is no published metabolomic analysis of plasma samples concerning response to anti-TNF-alpha treatment in RA patients.

\section{Conclusion}

This is the first study describing metabolic profiling in response to anti-TNF treatments using plasma samples.
The study highlighted two different metabolic profiles splitting good responders from non-responders. Our metabolomic approach needs to be completed by screening larger cohorts of patients and investigating outcomes with other biologic agents in patients with severe RA. Metabolomic analysis remains expensif but in case of identification of pertinent biomakers, classic quantitative analysis could be used in clinical practice.

\section{Acknowledgements}

The authors would like to thank Dihya Abdi, Angélique Fan and Thomas Frayssac and for editorial and administrative support.

\section{Funding}

This study was performed thanks to Hospital and Clinical Research Program (PHRC Interrégional, DGOS) and The Passerelle 2012 founding (Pfizer).

\section{Availability of data and materials}

The dataset supporting the conclusions presented in this article is available on request from the corresponding author.

\section{Authors' contributions}

ZT FG conceived of the study, and participated in its design and coordination and drafted the manuscript. CM carried out the matabolomic analysis and revised the manuscript. MP performed the statistical analysis and helped to revise the manuscript. EPG carried out the matabolomic analysis and revised the manuscript. PG participated in plasma bank's constitution and revised the manuscript. TL participated in plasma bank's constitution and revised the manuscript. HM participated in plasma bank's constitution and revised the manuscript. JT participated in plasma bank's constitution and revised the manuscript. MS conceived of the study and helped to draft the manuscript. All authors reviewed and approved the final manuscript.

\section{Competing interests}

The authors declare that they have no competing interests.

\section{Consent for publication}

Not applicable.

\section{Ethics approval and consent to participate}

The protocol for the study was approved by local institutional review boards or ethical review committees (Committee of Patient's Protection Grenoble 05-CHUG3 et Sud-Est 6 -AU731) and was conducted according to principles of Good Clinical Practice. Patients provided informed consent prior to participation in the study.

\section{Author details}

${ }^{1}$ Rheumatology Department, CHU Gabriel Montpied, 58 rue Montalembert B.P. 392, 63011 Clermont-Ferrand, France. ${ }^{2}$ Metabolomics Platform, INRA, Clermont-Ferrand, France. ${ }^{3}$ Rheumatology Department, CHU GrenobleHôpital Sud, Echirolles, France. ${ }^{4} \mathrm{CHU}$ Bois-Guillaume, Rouen, France. ${ }^{5}$ INSERM 1059, Université de Lyon, Saint-Etienne, France. ${ }^{6}$ Rheumatology Department, CHU Saint-Etienne, Saint-Etienne, France. ${ }^{7}$ Rheumatology Department, Centre Hospitalier Lyon-Sud, Pierre-Bénite, France.

Received: 19 December 2015 Accepted: 12 August 2016

Published online: 22 August 2016

\section{References}

1. Marotte $H$, Miossec P. Biomarkers for prediction of TNFalpha blockers response in rheumatoid arthritis. Joint Bone Spine. 2010;77(4):297-305.

2. Mugnier B, Roudier J. Factors predicting responsiveness to anti-TNFalpha therapy in patients with rheumatoid arthritis: using biotherapies rationally. Joint Bone Spine. 2004;71(2):91-4.

3. Conaghan PG. Predicting outcomes in rheumatoid arthritis. Clin Rheumatol. 2011;30 Suppl 1:S41-7.

4. Madsen $\mathrm{R}$, Lundstedt $\mathrm{T}$, Trygg J. Chemometrics in metabolomics-a review in human disease diagnosis. Anal Chim Acta. 2010;659(1-2):23-33. 
5. Arnett FC, Edworthy SM, Bloch DA, McShane DJ, Fries JF, Cooper NS, et al. The American Rheumatism Association 1987 revised criteria for the classification of rheumatoid arthritis. Arthritis Rheum. 1988;31:315-24.

6. Fries JF, Spitz $P$, Kraines RG, Holman HR. Measurement of patient outcome in arthritis. Arthritis Rheum. 1980;23:137-45.

7. Fransen J, Van Riel PL. The Disease Activity Score and the EULAR response criteria. Clin Exp Rheumatol. 2005;23:S93-9.

8. Van Gestel AM, Haagsma CJ, Riel PL. Validation of rheumatoid arthritis improvement criteria that include simplified joint counts. Arthritis Rheum. 1998:41:1845-50.

9. Harrell Jr FE, Lee KL, Mark DB. Multivariable prognostic models: issues in developing models, evaluating assumptions and adequacy, and measuring and reducing errors. Stat Med. 1996;15(4):361-87.

10. Cohen JE. Estimating the effects of successful malarial control programmes on mortality. Popul Bull UN. 1988;25:6-26.

11. Kapoor SR, Filer A, Fitzpatrick MA, Fisher BA, Taylor PC, Buckley CD, Mclnnes IB, Raza K, Young SP. Metabolic profiling predicts response to anti-tumor necrosis factor a therapy in patients with rheumatoid arthritis. Arthritis Rheum. 2013;65(6):1448-56.

12. Alavi A, Axford JS. Glyco-biomarkers: potential determinants of cellular physiology and pathology. Dis Markers. 2008;25(4-5):193-205.

13. Nimmerjahn F, Ravetch JV. Fc-receptors as regulators of immunity. Adv Immunol. 2007:96:179-204.

14. Alavi A, Axford JS. The pivotal nature of sugars in normal physiology and disease. Wien Med Wochenschr. 2006;156(1-2):19-33.

15. Nandakumar KS, Collin M, Olsén A, Nimmerjahn F, Blom AM, Ravetch JV, Holmdahl R. Endoglycosidase treatment abrogates lgG arthritogenicity: importance of lgG glycosylation in arthritis. Eur J Immunol. 2007;37(10):2973-82.

16. Axford JS, Cunnane G, Fitzgerald O, Bland JM, Bresnihan B, Frears ER. Rheumatic disease differentiation using immunoglobulin $\mathrm{G}$ sugar printing by high density electrophoresis. J Rheumatol. 2003;30(12):2540-6.

17. Jefferis R. Antibody therapeutics: isotype and glycoform selection. Expert Opin Biol Ther. 2007;7(9):1401-13.

18. Newkirk MM, Fournier MJ, Shiroky J. Rheumatoid factor avidity in patients with rheumatoid arthritis: identification of pathogenic RFs which correlate with disease parameters and with the gal(0) glycoform of lgG. J Clin Immunol. 1995;15(5):250-7.

19. Axford JS, Sumar N, Alavi A, Isenberg DA, Young A, Bodman KB, Roitt IM. Changes in normal glycosylation mechanisms in autoimmune rheumatic disease. J Clin Invest. 1992;89(3):1021-31.

20. Das H, Atsumi T, Fukushima Y, Shibuya H, Ito K, Yamada Y, Amasaki Y, Ichikawa K, Amengual O, Koike T. Diagnostic value of antiagalactosyllgGantibodies in rheumatoidarthritis. Clin Rheumatol. 2004;23(3):218-22.

21. vanZeben D, Rook GA, Hazes JM, Zwinderman AH, Zhang Y, Ghelani S, Rademacher TW, Breedveld FC. Early agalactosylation of lgG is associated with a more progressive disease course in patients with rheumatoid arthritis: results of a follow-up study. Br J Rheumatol. 1994;33(1):36-43.

22. Gindzienska-Sieskiewicz E, Klimiuk PA, Kisiel DG, Gindzienski A, Sierakowski S. The changes in monosaccharide composition of immunoglobulin $G$ in the course of rheumatoid arthritis. Clin Rheumatol. 2007;26(5):685-90.

23. Pasek M, Duk M, Podbielska M, Sokolik R, Szechiński J, Lisowska E, Krotkiewski H. Galactosylation of IgG from rheumatoid arthritis (RA) patients-changes during therapy. Glycoconj J. 2006;23(7-8):463-71.

24. Alavi A, Arden N, Spector TD, Axford JS. Immunoglobulin G glycosylation and clinical outcome in rheumatoid arthritis during pregnancy. J Rheumatol. 2000;27(6):1379-85.

25. Van Aken J, Lard LR, le Cessie S, Hazes JM, Breedveld FC, Huizinga TW. Radiological outcome after four years of early versus delayed treatment strategy in patients with recent onset rheumatoid arthritis. Ann Rheum Dis. 2004;63(3):274-9.

26. Lard LR, Visser $H$, Speyer I, van der Horst- Bruinsma IE, Zwinderman AH, Breedveld FC, et al. Early versus delayed treatment in patients with recentonset rheumatoid arthritis: comparison of two cohorts who received different treatment strategies. Am J Med. 2001;111(6):446-51.

27. Nell VPK, Machold KP, Eberl G, Stamm TA, Uffmann M, Smolen JS. Benefit of very early referral and very early therapy with disease- modifying antirheumatic drugs in patients with early rheumatoid arthritis. Rheumatology. 2004;43(7):906-14.

28. Weljie AM, Dowlatabadi R, Miller BJ, Vogel HJ, Jirik FR. An inflammatory arthritis-associated metabolite biomarker pattern revealed by $1 \mathrm{H}$ NMR spectroscopy. J Proteome Res. 2007;6(9):3456-64.
29. Madsen RK, Lundstedt T, Gabrielsson J, Sennbro CJ, Alenius GM, Moritz T, Rantapää-Dahlqvist S, Trygg J. Diagnostic properties of metabolic perturbations in rheumatoid arthritis. Arthritis Res Ther. 2011;13(1):R19.

30. Lauridsen MB, Bliddal H, Christensen R, Danneskiold-Samsøe B, Bennett R, Keun H, Lindon JC, Nicholson JK, Dorff MH, Jaroszewski JW, Hansen SH, Cornett C. 1H NMR spectroscopy-based interventional metabolic phenotyping: a cohort study of rheumatoid arthritis patients. J Proteome Res. 2010;9(9):4545-53.

31. Seeger K. Metabolic changes in autoimmune diseases. Curr Drug Discov Technol. 2009;6(4):256-61.

32. Um SY, Chung MW, Kim KB, Kim SH, Oh JS, Oh HY, Lee HJ, Choi KH. Pattern recognition analysis for the prediction of adverse effects by nonsteroidal anti-inflammatory drugs using $1 \mathrm{H}$ NMR-based metabolomics in rats. Anal Chem. 2009:81(12):4734-41.

\section{Submit your next manuscript to BioMed Central and we will help you at every step:}

- We accept pre-submission inquiries

- Our selector tool helps you to find the most relevant journal

- We provide round the clock customer support

- Convenient online submission

- Thorough peer review

- Inclusion in PubMed and all major indexing services

- Maximum visibility for your research

Submit your manuscript at www.biomedcentral.com/submit
Biomed Central 